\title{
Genome-wide DNA methylation profiling reveals methylation markers associated with $3 q$ gain for detection of cervical pre-cancer and cancer
}

Wina Verlaat ${ }^{1}$, Peter J.F. Snijders ${ }^{1}$, Putri W. Novianti ${ }^{1,2}$, Saskia M. Wilting ${ }^{1}$, Lise M.A. De Strooper ${ }^{1}$, Geert Trooskens ${ }^{3}$,Johan Vandersmissen ${ }^{3}$, Wim Van Criekinge ${ }^{3}$, G. Bea A. Wisman ${ }^{4}$, Chris J.L.M. Meijer $^{1}$, Daniëlle A.M. Heideman ${ }^{1}$, Renske D.M. Steenbergen ${ }^{1 *}$

${ }^{1}$ Department of Pathology, VU University Medical Center, Amsterdam, The Netherlands ${ }^{2}$ Department of Epidemiology and Biostatistics, VU University Medical Center, Amsterdam, The Netherlands

${ }^{3}$ Department of Mathematical Modeling, Statistics and Bioinformatics, Ghent University, Ghent, Belgium.

${ }^{4}$ Department of Gynecologic Oncology, Cancer Research Center Groningen, University of Groningen, University Medical Center Groningen, Groningen, The Netherlands

\section{${ }^{*}$ Corresponding author:}

Renske D.M. Steenbergen, PhD, Department of Pathology, VU University Medical Center, PO Box 7057, 1007 MB Amsterdam; The Netherlands, E-mail: $\underline{\text { r.steenbergen@vumc.nl }}$

Running title:

Methylation markers at $3 q$ for cervical (pre)cancer detection

Key words:

Methyl Binding Domain/MBD-Seq, epigenetics, copy number aberrations, cervical carcinogenesis, biomarkers

This article was published in Clin Cancer Res. 2017; doi: 10.1158/1078-0432.CCR-16-2641. The published article PDF can be found at:

http://clincancerres.aacrjournals.org/content/early/2017/01/24/1078-0432.CCR-16-2641.full$\underline{\text { text.pdf }}$ 


\section{Translational relevance}

Implementation of primary high-risk HPV (hrHPV) DNA testing in cervical screening programs in the coming years results in better protection against cervical (pre)cancer. However, its specificity for clinically meaningful disease is lower compared to current cytology-based screening. Furthermore, current methods are unable to determine which precursor lesions will progress or regress, leading to over-referral and overtreatment. This study aimed to identify biologically relevant DNA methylation changes that enable identification of hrHPV-positive women with clinically relevant cervical lesions who are in need of referral. Following an unbiased genome-wide approach, we identified 3 novel biologically relevant methylation markers (GHSR, SST and ZIC1, all located on chromosome 3q), that provide most promising molecular triage markers for detection of cervical (pre)cancer in cervical scrapes. These methylation markers mainly detect advanced precursor lesions with a high likelihood of short-term progression risk to cancer, leading to detection of clinically relevant precursor lesions. These findings could greatly advance the management of hrHPV-positive women. 


\begin{abstract}
Purpose

Epigenetic host cell changes involved in cervical cancer development following a persistent high-risk human papillomavirus (hrHPV) infection, provide promising markers for the management of hrHPVpositive women. In particular, markers based on DNA methylation of tumour suppressor gene promoters are valuable. These markers ideally identify hrHPV-positive women with pre-cancer $(\mathrm{CIN} 2 / 3)$ in need of treatment. Here, we set out to identify biologically relevant methylation markers by genome-wide methylation analysis of both hrHPV-transformed cell lines and cervical tissue specimens.
\end{abstract}

\title{
Experimental Design and Results
}

Genome-wide discovery by next generation sequencing (NGS) of Methyl Binding Domain enriched DNA (MBD-Seq) yielded 20 candidate methylation target genes. Further verification and validation by multiplex targeted bisulphite NGS and (quantitative) methylation-specific PCR (MSP) resulted in 3 genes (GHSR, SST and ZIC1) that showed a significant increase in methylation with severity of disease in both tissue specimens and cervical scrapes $(p<0.005)$. The area under the ROC curve for CIN3 or worse varied between $0.86-0.89$. Within the group of $\mathrm{CIN} 2 / 3$, methylation levels of all 3 genes increased with duration of lesion existence $(p<0.0005)$, characterized by duration of preceding hrHPV infection, and were significantly higher in the presence of a $3 q$ gain $(p<0.05)$ in the corresponding tissue biopsy.

\section{Conclusion}

By unbiased genome-wide DNA methylation profiling and comprehensive step-wise verification and validation studies using in vitro and patient-derived samples, we identified 3 promising methylation markers (GHSR, SST and ZIC1) associated with a 3q gain for the detection of cervical (pre)cancer. 


\section{Introduction}

Cervical cancer is caused by a persistent high-risk human papillomavirus (hrHPV) infection, with HPV16 and HPV18 being the most predominant types(1). Cervical squamous cell carcinoma (SCC) develops through well-defined precursor lesions (cervical intraepithelial neoplasia, classically graded 1 to 3 (CIN1-3)), of which CIN3 is considered the most advanced precursor lesion of cervical cancer(2-4). Common practice is the ablation of CIN2 and CIN3 tissue to prevent cervical cancer development. However, many CIN2 and some CIN3 lesions will regress spontaneously if left untreated $(2,3,5)$. Current CIN classification is not able to identify which lesions will progress or regress, leading to considerable over-referral and overtreatment with associated cervical morbidity. The progression of CIN2/3 to SCC results from the accumulation of genetic and epigenetic alterations in the host cell over a time period of 15 to 30 years(2-4). Given their variable duration of existence, CIN2/3 represents a heterogeneous disease, with various risk of progression to cancer. Recent molecular studies on $\mathrm{CIN} 2 / 3$ indicate that a subset of these lesions display a cancer-like profile including hypermethylation of tumour suppressor genes and specific chromosomal alterations(6-8). These so-called advanced CIN2/3 lesions, characterized by a longer duration ( $\geq 5$ years) of preceding hrHPV infection (PHI), are most likely to have a high short-term progression risk to cancer and are in need of treatment(2). DNA methylation-based assays that can be used as a biomarker for the detection of advanced CIN2/3 lesions would greatly advance the management of hrHPV-positive women by reducing overdiagnosis and overtreatment.

At present, a number of methylated host cell genes have been described by us and others as promising markers for the management of hrHPV-positive women, such as CADM1, MAL, miR124-2, FAM19A4 and EPB41L3(7,9-12). Here, we describe an unbiased genome-wide based approach, comprised of next generation sequencing (NGS) of Methyl Binding Domain (MBD) enriched DNA (MBD-Seq), to identify biologically relevant methylation-based markers within patient-derived and in vitro samples representing the multistep process of hrHPV-induced carcinogenesis. Our longitudinal in vitro model of primary keratinocytes immortalized by hrHPV types closely mimics in vivo carcinogenic stages, both with respect to molecular as well as morphological changes(13). This model system allows us to study the sequential order of accumulating epigenetic host cell changes associated with hrHPV-mediated carcinogenesis(14-16). Combined with the DNA methylation profiles of various stages of cervical (pre)cancer in vivo, this offers a rewarding strategy to identify biologically relevant methylation markers. Additionally, step-wise verification and validation studies were performed using multiplex targeted bisulphite NGS, methylation-specific PCR (MSP) and multiplex quantitative MSP (qMSP) on large clinical series of both cervical tissues and scrapes, comprising all stages of cervical carcinogenesis. To further understand the relation of DNA methylation levels in cervical scrapes to the severity and duration of the underlying cervical lesion, samples from women with $\mathrm{CIN} 2 / 3$ with a known duration of $\mathrm{PHI}$, as a proxy of lesion duration, were included(6). Furthermore, to link methylation findings to other critical host cell alterations such as DNA copy number aberrations, we analysed previously obtained chromosomal profiles of these samples(6). Altogether, our comprehensive approach yielded 3 novel promising methylation markers associated with $3 q$ gain for the detection of cervical (pre)cancer. 


\section{Materials and Methods}

\section{Cell lines and clinical specimens}

\section{Discovery}

For genome-wide DNA methylation profiling by MBD-Seq, various cell lines and frozen cervical tissue specimens were used. Cell lines consisted of (i) primary human foreskin keratinocytes (HFK) from two donors, (ii) HFK from one donor transduced with HPV16E6E7 at passage 7 and passage 30, representing the pre-immortal and immortal stage, respectively(17), (iii) consecutive passages of HPV16- (FK16A and FK16B) and HPV18- (FK18A and FK18B) immortalized keratinocytes, including early (passages 11-30), middle (passages 30-70) and late (>passage 70) immortal passages, and (iv) cervical cancer cell line SiHa. The tissue specimens comprised CIN1 ( $n=3), \mathrm{CIN} 2 / 3$ ( $n=12)$ and SCC $(n=10)$. Given limitations in accurate histological grading of high-grade CIN on frozen tissue sections, CIN2 and CIN3 were grouped together in this frozen tissue sample series.

\section{Verification I}

Multiplex targeted bisulphite NGS was performed on a subset of hrHPV-transformed cell lines and frozen tissue specimens used for discovery with sufficient material left, i.e., HFKs from two donors, early and late immortal passages of FK16B and FK18B, SiHa, CIN2/3 lesions ( $n=8)$ and SCCs $(n=7)$. In addition, 2 pooled control specimens comprising pooled DNAs of 3 normal cervical formalin-fixed paraffin-embedded (FFPE) tissue specimens or 6 scrapes from women without cervical disease, respectively, were used.

\section{Verification II}

For verification by MSP, HFKs from four different donors, late immortal passages of FK16A, FK16B and FK18A, cervical cancer cell lines SiHa, HeLa and CaSki, and FFPE tissue specimens including normal cervical tissue $(n=11)$, CIN3 $(n=11)$ and SCC $(n=10)$ were used.

\section{Validation}

For validation by multiplex qMSP, HFKs from five different donors, middle and late immortal passages of FK16A, FK16B, FK18A and FK18B, and cervical cancer cell lines SiHa, HeLa and CaSki were used. In addition, an independent series of FFPE tissue specimens comprising normal cervical tissue $(n=41), \operatorname{CIN} 1(n=32), \operatorname{CIN} 2(n=23), \operatorname{CIN} 3(n=33)$ and SCC $(n=30)$, as well as a series of scrapes comprising specimens from hrHPV-positive women with normal cytology and/or without evidence of $\mathrm{CIN} 2+(n=97)$ or from women who were histologically diagnosed with CIN2 ( $n=21), \operatorname{CIN} 3(n=75)$ or SCC $(n=27)$, were used. Another 48 cervical scrapes from women with a CIN2 or CIN3 lesion in the second screening round (interval 5 years) of the POBASCAM trial(18)were included. Details of this additional series have been described before(6-8). In brief, the 5-year history of hrHPV infection of these women was known and used as surrogate for duration of lesion existence. Women with a PHI $<5$ years were considered to have early $\mathrm{CIN} 2 / 3$ lesions $(n=19)$ and women with a $\mathrm{PHI} \geq 5$ years were considered to have advanced $\mathrm{CIN} 2 / 3$ lesions $(n=29)$. DNA copy number data were obtained during a previous study in which tissue specimens corresponding to this series of cervical scrapes were microdissected to enrich for the dysplastic areas and isolated DNA was hybridised to $105 \mathrm{~K}$ arrays (Agilent Technologies, Palo Alto, USA)(6). DNA copy number data are available from the Gene Expression Omnibus (GEO, http://www.ncbi.nlm.nih.gov/projects/geo/), through series accession number GSE30155.

HFKs were obtained and cultured as described before(19). Cervical cancer cell lines SiHa, HeLa and CaSki (American Type Culture Collection) were cultured according to specifications(20). Cell lines were recently authenticated using the PowerPlex 16 System (Promega, Madison, WI, USA) and were negative for mycoplasma. The establishment and culturing of HFKs transduced with HPV16E6E7 as well as FK16A, FK16B, FK18A and FK18B have been described previously $(17,19)$. All biopsies were 
collected during the course of routine clinical practice at the Department of Obstetrics and Gynaecology at VU University Medical Center, Amsterdam, The Netherlands. Cervical scrapes were obtained from screening or gynaecologic outpatient populations. This study followed the ethical guidelines of the Institutional Review Board of the VU University Medical Center and University Medical Center Groningen.

\section{DNA isolation, bisulphite treatment and hrHPV testing}

Isolation of DNA from cell lines was performed by proteinase $K$ digestion followed by standard phenol-chloroform extraction as described previously(21). Isolation of DNA from tissue specimens was performed by High Pure PCR Template Preparation Kit (Roche Diagnostics, Basel, Switzerland) using manufacturer's protocol. DNA from cervical scrapes was isolated using the NucleoMag 96 Tissue kit (Macherey-Nagel, Düren, Germany) and a Microlab Star robotic system (Hamilton, Martinsried, Germany) according to manufacturer's protocol. For multiplex targeted bisulphite NGS, MSP and qMSP analyses, DNA was bisulphite-converted using the EZ DNA Methylation kit (Zymo Research, Orange, CA, USA). HrHPV DNA detection was performed by GP5+/6+ PCR enzyme immunoassay or HPV-Risk assay (Self-screen BV, Amsterdam, The Netherlands)(22,23).

\section{MBD-Seq}

Genome-wide DNA methylation profiling was performed by MBD-Seq, using the MethylCap kit v2 (Diagenode, Liège, Belgium) with 500 ng input DNA combined with Illumina Genome Analyzer IIx paired-end sequencing as described before $(24,25)$. Next, Bowtie 1.0 .0 was used to map the obtained paired-end reads from fastq-files to the human reference genome GRCh37(26). These mapped sequencing results were visualized in the $\mathrm{H} 2 \mathrm{G} 2$ genome browser (http://h2g2.ugent.be/biobix.html). From this browser, we extracted the read counts of all predefined methylated regions located in promoters ( $-1000 \mathrm{bp}$ to $+500 \mathrm{bp}$ relative to the transcription start site). The read counts of all regions in cervical cancer ( $\mathrm{SiHa}, \mathrm{SCC}$ ) were compared to the read counts in controls (HFK, CIN1). These regions were ranked based on the difference in read counts in order to select the hypermethylated regions. Next, we analysed hypermethylation of these regions in the precursors (HFKs transduced with HPV16E6E7, FK16A, FK16B, FK18A, FK18B and CIN2/3). From this list, we selected the top 20 most hypermethylated promoters.

\section{Multiplex targeted bisulphite NGS}

For multiplex targeted bisulphite NGS, a total of 62 primer pairs were designed to amplify the 20 selected methylated regions by PCR with on average 3 amplicons per target. Each forward and reverse primer contained a universal extension. The amplicons were pooled together per sample and purified from primer dimers using the Gel Extraction kit (Qiagen, Hilden, Germany). A second PCR was performed on the pool of amplicons using primers annealing to the universal extension to add the flow cell binding sites $p 5$ and $p 7$ and a unique barcode to every sample. The concentration of every sample library was measured using the Bioanalyzer 2100 (Agilent Technologies, Santa Clara, CA, USA). Up to 12 uniquely barcoded sample libraries were equimolarly pooled prior to sequencing. This final pool contained 10\% spike-in of PhiX Control v3 (Illumina, San Diego, CA, USA) to increase the diversity and was loaded on a MiSeq Personal Sequencer (Illumina) using 5 pM. A MiSeq Reagent Kit v2 (300 cycles; Illumina) was used in a paired-end 150 run according to manufacturer's protocol. All bisulphite sequencing data described in this study were processed as follows: Quality was evaluated using FastQC Version 0.11.2, the reads were aligned on the bisulphite treated version of the human genome and methylation calls were performed in Bismark version 0.12.9(27). Reads were combined to represent a DNA methylation profile for each sample.

\section{MSP and multiplex qMSP}

MSP was performed as previously described using $50 \mathrm{ng}$ of bisulphite-converted DNA as input material(28). MSP primers were designed to specifically amplify methylated bisulphite treated DNA. In vitro methylated DNA (IVD) was used as positive control and unmodified SiHa DNA and $\mathrm{H}_{2} \mathrm{O}$ served 
as negative controls. The housekeeping gene $\beta$-actin (ACTB) verified sufficient DNA quality and successful bisulphite conversion of all samples.

Multiplex qMSP for 3 targets and ACTB was designed as described before(29), and performed using $50 \mathrm{ng}$ of bisulphite-converted DNA and 200-300 nM of each primer and fluorescent dyelabelled probe, on the ABI 7500 Fast Real-Time PCR System (Applied Biosystems, Foster City, CA, USA). The methylation values of the targets were normalised to reference gene $A C T B$ using the comparative $\mathrm{Cq}$ method $\left(2^{-\Delta C q} \times 100\right)$ to obtain Cq ratios. All samples in qMSP analysis had an ACTB Cq value $<32$, indicating suitability for DNA methylation analysis.

\section{Statistical analyses}

For each methylated gene, Wilcoxon rank test was applied to test the difference in methylation levels between disease categories with a significance level of 0.05 (two-sided). For logistic regression analysis in cervical scrapes, samples from women with CIN3 or SCC (CIN3+) were categorized as cases and those from hrHPV-positive women with normal cytology and/or without evidence of CIN2+ were categorized as controls. To assess the relationship between the outcome of the samples (case or control) and the methylation markers, univariable logistic regression analysis was performed on the square root transformed Cq ratios of GHSR, SST and ZIC1. The performance of the logistic regression model was visualized by receiver operating characteristics (ROC) curve, and was evaluated by sensitivity, specificity and area under the curve (AUC). Predicted probabilities, representing the risk for an underlying CIN3+, were calculated using the univariable logistic regression models of GHSR, SST and ZIC1. Samples with predicted probabilities above the optimum threshold (GHSR: 0.396; SST: 0.358; ZIC1: 0.354) were scored methylation-positive for the respective marker. 


\section{Results}

An outline of the study is given in Figure 1 and Supplementary Table S1.

\section{Genome-wide DNA methylation profiling by MBD-Seq}

For discovery of DNA methylation profiles associated with hrHPV-induced carcinogenesis, genomewide MBD-Seq was performed on primary keratinocytes, hrHPV-transformed cell lines and cervical tissue specimens (Figure 1; Discovery). Differentially methylated promoter regions were identified as those with a difference in cervical cancer samples (SiHa, SCC) over normal/low-grade samples (HFK, CIN1), and precursors (hrHPV-transformed cell lines and CIN2/3 lesions) over normal/low-grade samples. Differentially methylated promoters were ranked to select the top 20 candidate hypermethylation target genes (Table 1).

\section{Verification analyses and selection of candidate methylation markers}

Verification of the top 20 candidates by multiplex targeted bisulphite NGS was performed on a subset of samples used in the MBD-Seq screen (Figure 1; Verification I). Increased methylation of 9 genes, GALR1, GHSR, IRX1, NEUROG3, POU4F3, PROX1, SIM2, SST and ZIC1, was verified in hrHPVimmortalized keratinocytes, cervical cancer cells, CIN2/3 lesions and SCCS compared to controls (Table 1). The remaining 11 genes were excluded from further studies due to the detection of methylation in controls (ANKRD2, CLDN18, GRK7, HIPK4, SLC45A2, TBX19 and TFDP3) or technical issues (MYCL2, PDE4DIP, RXFP3 and SOX14) (Table 1).

Subsequent MSP analysis confirmed methylation of all 9 genes in late immortal passages of hrHPV-immortalized keratinocytes and cervical cancer cell lines, while HFKs were mainly methylation-negative (Figure 1; Verification II; Table 1, Supplementary Fig. S1A). SIM2 was clearly methylation-positive in 1 out of 4 HFKs. GALR1, IRX1 and NEUROG3 were ambiguously methylationpositive in some late immortal passages of hrHPV-immortalized keratinocytes. The remaining five genes (GHSR, POU4F3, PROX1, SST and ZIC1) were further evaluated by MSP in a series of cervical tissue specimens (Figure 1; Verification II) confirming increased methylation frequency with increasing disease severity (Table 1, Supplementary Fig. S1B and Fig. S2). Two genes, POU4F3 and PROX1, were methylation-negative in 1 out of 10 SCCs leaving 3 genes (GHSR, SST and ZIC1) for a comprehensive validation analysis.

\section{Validation of candidate markers in cell lines and cervical tissue specimens by qMSP analysis}

Based on the verification findings, GHSR, SST and ZIC1, were further analysed by multiplex qMSP on cell lines and independent large series of cervical tissue specimens (Figure 1; Validation). No SST and ZIC1 methylation was detected in any of the HFKs, whereas GHSR showed very low levels of methylation in 3 out of 5 HFKs. Methylation of all 3 genes significantly increased with passaging of hrHPV-immortalized keratinocytes and was highest in cervical cancer cell lines ( $p<0.05$; Figure $2 A-C)$. For all 3 genes, a gradual increase in methylation was observed with increasing severity of cervical disease. Methylation levels were significantly increased in CIN2 compared to normal cervical tissues ( $p<0.005)$, in CIN3 compared to CIN2 ( $p<0.05)$, as well as in SCCs compared to CIN3 ( $<<0.0005$; Figure 2D-F).

\section{Marker potential of GHSR, SST and ZIC1 in cervical scrapes}

Next, validation analysis included multiplex qMSP on a total of $220 \mathrm{hrHPV}$-positive cervical scrapes to evaluate the potential of GHSR, SST and ZIC1 methylation analysis to detect cervical (pre)cancer (Figure 1; Validation). For all 3 genes, fold changes in methylation levels compared to the reference group (hrHPV-positive controls) increased with severity of underlying disease (Table 2A). Largest fold changes were seen for ZIC1, showing a 10.1-fold increase in scrapes from women with CIN2, elevating to a 58.7-fold change in scrapes from women with CIN3, and a 921.1 fold-change in scrapes from women with SCC. Differences were significant for all 3 genes in hrHPV-positive scrapes from 
women with CIN3+ compared to hrHPV-positive controls $(\mathrm{p}<0.0005)$ and hrHPV-positive scrapes from women with CIN2 ( $p<0.005$; Supplementary Fig. S3). Logistic regression analysis showed a similar clinical performance for CIN3+ detection in hrHPV-positive cervical scrapes for GHSR, SST and ZIC1, with an AUC of 0.87, 0.86 and 0.89 , respectively (Figure 3A; Table 3). ZIC1 presented the highest AUC, which corresponds at a certain threshold to a sensitivity of $86.3 \%$ and a specificity of $80.4 \%$ (Table 3 ). All cervical cancers were detected in scrapes by GHSR, whereas SST and ZIC1 detected identical 26 of $27(96.3 \%)$ cervical cancers.

Methylation levels of GHSR, SST and ZIC1 in cervical scrapes from women with CIN2/3 lesions with different duration of existence and known DNA copy number profile

To evaluate marker potential in relation to advanced CIN2/3, cervical scrapes from women with an underlying $\mathrm{CIN} 2 / 3$ lesion with a known duration of $\mathrm{PHI}$, reflecting duration of lesion existence (19 early CIN2/3 lesions: $<5$ year PHI; 29 advanced CIN2/3 lesions: $\geq 5$ year PHI) were analysed by multiplex qMSP (Figure 1; Validation). Methylation levels of GHSR, SST and ZIC1 increased with duration of PHI. Methylation levels of GHSR, SST and ZIC1 were significantly increased in cervical scrapes from women with advanced CIN2/3 compared to scrapes from women with early $\mathrm{CIN} 2 / 3$ $(p<0.0005$; Figure 3B-D), showing a 13.3-, 9.7- and 47.1-fold increase, respectively, over the reference (Table 2B), with methylation levels in scrapes from women with early CIN2/3 being comparable to the reference group. Using the above described logistic regression model, most cervical scrapes from women with advanced CIN2/3 scored methylation-positive by GHSR (20/29; 69\%), SST (17/29; 58.6\%) and ZIC1 (20/29; 69\%), whereas all (SST and ZIC1) or majority (GHSR; 18/19; 94.7\%) of scrapes from women with early CIN2/3 were methylation-negative. A combination of GHSR and ZIC1, showing methylation-positivity for at least one of the markers, increased the detection of advanced $\mathrm{CIN} 2 / 3$ (22/29; 75.9\%).

As GHSR, SST and ZIC1 are all located on chromosome 3q, a region that is gained in $55 \%$ of cervical SCC cases(30), we additionally evaluated a potential association of methylation levels with gain of the gene locus. For this purpose, copy number data obtained during previous studies on the underlying advanced and early $\mathrm{CIN} 2 / 3$ lesions were used(6). Methylation levels of all 3 genes in the cervical scrapes were significantly higher in the presence of a $3 q$ gain in the corresponding $\mathrm{CIN} 2 / 3$ lesions compared to the absence of a $3 q$ gain $(p<0.05$; Figure $3 E-G)$, which was also reflected by their fold changes over the reference group (i.e. 50.9-153 in presence of $3 q$ gain versus 2.30-29.6 in absence of 3q gain; Table 2B). For comparison, previously obtained methylation levels of FAM19A4 (chr. 3p14.1), CADM1 (chr. 11q23.2), MAL (chr. 2q11.1) and miR124-2 (chr. 8q12.3)(7,8) were not significantly different between scrapes from women with advanced CIN2/3 with and without a $3 q$ gain (Supplementary Fig. S4). 


\section{Discussion}

By unbiased genome-wide DNA methylation profiling and subsequent comprehensive step-wise verification and validation studies using in vitro and patient-derived samples representing the multistep process of hrHPV-induced carcinogenesis, we identified 3 new promising methylation markers (GHSR, SST and ZIC1) associated with 3q gain for the detection of cervical (pre)cancer. Methylation levels of GHSR, SST and ZIC1 showed a significant increase with severity of disease, reaching high levels in advanced $\mathrm{CIN} 2 / 3$ and SCC, both when analysing tissue specimens as well as cervical scrapes. Within the group of women with $\mathrm{CIN} 2 / 3$ lesions, methylation levels of all 3 genes in cervical scrapes increased with duration of lesion existence $(p<0.0005)$ and were significantly higher in the presence of a $3 q$ gain $(p<0.05)$ in the corresponding tissue biopsy. Logistic regression analysis of GHSR, SST and ZIC1 in hrHPV-positive cervical scrapes for CIN3+ detection resulted in an AUC of $0.87,0.86$ and 0.89 , respectively, showing a similar and good clinical performance of all 3 genes, supporting the potential of these methylation markers for the management of hrHPV-positive women. In contrast to cytology-based triage tests, such as p16/Ki-67 dual-stained cytology, methylation marker analysis has an improved reliability and it is applicable on both cervical scrapes and self-collected cervical-vaginal specimens. Methylation marker analysis may also be combined with HPV16/18 genotyping, and has been found complementary in detection of CIN2/3 and cancer. DNA methylation is especially sensitive for detection of cancer and advanced CIN2/3, whereas most early CIN2/3 are detected by HPV16/18 genotyping(31). Additionally, these 3 markers could potentially also be useful for early detection of other HPV-associated cancer types, such as a subset of head and neck cancers and other anogenital cancers.

The advantages of our study are the evaluation of large, independent series of different in vitro and patient-derived sample types and the use of a systematic discovery-verification-validation approach. MBD-Seq is an effective discovery methodology for biologically relevant methylation markers as it enriches for methylation targets characterized by methylation of multiple consecutive CpGs, which is a characteristic of gene silencing when affecting promoter regions, and an advantage over commonly used techniques detecting only methylation of single $\mathrm{CpGs}(32,33)$. Furthermore, using MBD-Seq we could also detect methylated HPV DNA by analysis of the non-aligned reads(24). In the current study, both in vitro and patient-obtained samples comprising all stages of cervical carcinogenesis were used, which allowed us to evaluate the succession of methylation events and the selection of methylation targets which are biologically relevant and associated with hrHPVmediated carcinogenesis. Furthermore, by using both sample types during step-wise verification and validation, we could exclude candidates showing methylation very early or relatively late during carcinogenesis, and accordingly would result in unfavorable clinical specificity or sensitivity, respectively.

High-grade CIN lesions (CIN2/3) represent a heterogeneous stage of disease, only a subset of which may progress to cancer over a period of 15 to 30 years $(2,3)$. Previous data have shown that the heterogeneity in duration of lesion existence is reflected by their molecular profile, with significant increased copy number alterations and methylation levels in advanced CIN2/3 lesions compared to early $\mathrm{CIN} 2 / 3$ lesions(6-8). In line with previous findings(7,8), present data show that the methylation levels of GHSR, SST and ZIC1 are significantly increased in cervical scrapes from women with advanced CIN2/3 lesions compared to scrapes from women with early CIN2/3 lesions, which is also reflected in their frequency of methylation-positivity. Of note and in addition to previous findings, the current study shows that methylation of all 3 genes, which are located at chromosome $3 q$, i.e., GHSR (3q26), SST (3q28) and ZIC1 (3q24), is related to the presence of a 3q gain. Gain at 3q, which is the most frequently gained region in cervical cancer $(6,30)$, as well as amplification of the human telomerase gene (TERC; 3q26), have been suggested as a marker for progression to cancer $(34,35)$. The observation that methylation levels of genes at other chromosomal locations were not significantly increased in cervical scrapes from women with advanced $\mathrm{CIN} 2 / 3$ with a $3 q$ gain, suggests 
that DNA methylation of GHSR, SST and ZIC1 may be biologically important to compensate for increased gene dosage during cervical carcinogenesis.

At present, there are only a few studies which investigated a relation between DNA methylation and DNA copy number profiles in cervical cancer, and those were mostly focussed on allelic losses, such as for chromosomes $11 q$ and $3 p(20)$. In lung cancer, a correlation between DNA hypermethylation and gene amplification has been described for SHOX2, which is also located on chromosome $3 q(36)$. Another study described a significant association between DNA methylation of the HOXA locus and a gain of chromosome 7 in glioblastoma(37). MBD-Seq on cervical specimens by Boers et al., reported 15 candidate methylation targets(38), part of which were also located on known copy number gains, i.e. ST6GALNAC5 (1p), LHX8 (1p), CDH6 (5p) and IRX1 (5p). Yet, it is unclear whether DNA hypermethylation is a response reaction to copy number alterations or a cause of copy number alterations due to destabilization of the genome. Further studies are warranted to investigate the co-evolution of DNA methylation and copy number alterations, in particular copy number gains, using integrated copy number and methylation analysis. In the current study, we were unfortunately unable to acquire information on copy numbers from our sequencing data due to varying read-counts between samples using MBD-Seq analysis.

To the best of our knowledge, methylation of GHSR and ZIC1 has not been described before in cervical cancer, whereas SST was identified previously by Ongenaert et al(39). GHSR is the growth hormone secretagogue receptor, or ghrelin receptor, which regulates growth hormone secretion and functions in energy homeostasis(40). Methylation of GHSR has been described before in lung cancer, breast cancer, glioblastoma and B cell chronic lymphocytic leukaemia(41). ZIC1, zinc finger of the cerebellum family member 1 , functions as a transcription factor and has an important role in central nervous system, muscle and skeletal development(42). Methylation of ZIC1 has been described previously for other tumour types, such as thyroid, ovarian, colon and gastric cancer(43-46). SST or somatostatin functions as an inhibitor with anti-secretory, anti-proliferative and anti-angiogenic effects(47) and is, in addition to cervical cancer, also found to be methylated in gastric, esophageal, colon and head and neck cancer(48-51). The biological function and expression regulation of these 3 genes in HPV-transformed cells remains to be determined. Based on our in-house and publically available expression profiling data(52) of cervical tissue specimens and cell lines no firm conclusions can be drawn on their expression regulation as expression levels were relatively low in control samples. Likewise, differential protein expression was not detected in the Human Protein Atlas (http://www.proteinatlas.org/), since both cervical cancer tissue and normal tissue showed low or non-detectable protein levels.

In conclusion, using an unbiased genome-wide DNA methylation profiling approach and subsequent step-wise verification and validation studies on hrHPV-transformed cell lines and clinical cervical specimens, we identified 3 methylation markers, GHSR, SST and ZIC1, associated with $3 q$ gain, for detection of cervical (pre)cancer. Our findings warrant further clinical validation of GHSR, SST and ZIC1 in a prospective study to verify their potential as biomarkers for the management of hrHPV-positive women. 


\section{Acknowledgements}

We thank Marjolein van Zummeren, Hanne Sørgård, Annina van Splunter, Daoud Sie, Paul Eijk, Dirk van Essen, Larissa Vork and Tine Beyaert for excellent technical assistance.

\section{Financial support:}

This study was sponsored by Eurostars E!6679 Cervix-care and the European Research Council (ERC advanced 2012- AdG, proposal 322986; Mass-Care). The sources of funding did not have any influence on the design of the study, collection, analysis and interpretation of the data and in writing the manuscript.

\section{Conflict of interest:}

P. Snijders, C. Meijer, R. Steenbergen and D. Heideman have minority stake in Self-screen B.V., a spinoff company of VU University Medical Center Amsterdam, which owns patents related to this work. P. Snijders has been on the speaker's bureau of Roche, Abbott, Gen-Probe, Qiagen and Seegene. He is consultant for Crucell Holland B.V. C. Meijer has participated in the sponsored speaker's bureau of Merck, GSK, Qiagen, Menarini, Seegene, and Roche, and served occasionally on the scientific advisory board of GSK, Qiagen, Merck, and Roche. C. Meijer has occasionally been a consultant for Qiagen and Genticel and was a minority shareholder of Diassay B.V till March 2016 and of Delphi Biosciences till 2014. D. Heideman serves occasionally on the scientific advisory boards of Amgen and Pfizer. W. Van Criekinge has a relationship with MDxHealth, Irvine, CA, USA. G. Wisman holds patents related to the work. All other authors have no conflict of interest to declare. 


\section{Reference list}

1. Walboomers JMM, Jacobs M V, Manos MM, Bosch FX, Kummer JA, Shah K V, et al. Human papillomavirus is a necessary cause of invasive cervical cancer worldwide. J Pathol. 1999;189:12-9.

2. Steenbergen RDM, Snijders PJF, Heideman DAM, Meijer CJLM. Clinical implications of (epi)genetic changes in HPV-induced cervical precancerous lesions. Nat Rev Cancer. 2014;14:395-405.

3. McCredie MRE, Sharples KJ, Paul C, Baranyai J, Medley G, Jones RW, et al. Natural history of cervical neoplasia and risk of invasive cancer in women with cervical intraepithelial neoplasia 3: a retrospective cohort study. Lancet Oncol. 2008;9:425-34.

4. Vink MA, Bogaards JA, Van Kemenade FJ, De Melker HE, Meijer CJLM, Berkhof J. Clinical progression of high-grade cervical intraepithelial neoplasia: Estimating the time to preclinical cervical cancer from doubly censored national registry data. Am J Epidemiol. 2013;178:11619.

5. Trimble CL, Piantadosi S, Gravitt P, Ronnett B, Pizer E, Elko A, et al. Spontaneous regression of high-grade cervical dysplasia: Effects of human papillomavirus type and HLA phenotype. Clin Cancer Res. 2005;11:4717-23.

6. Bierkens M, Wilting SM, van Wieringen WN, van Kemenade FJ, Bleeker MCG, Jordanova ES, et al. Chromosomal profiles of high-grade cervical intraepithelial neoplasia relate to duration of preceding high-risk human papillomavirus infection. Int J Cancer. 2012;131:579-85.

7. De Strooper LMA, Meijer CJLM, Berkhof J, Hesselink AT, Snijders PJF, Steenbergen RDM, et al. Methylation analysis of the FAM19A4 gene in cervical scrapes is highly efficient in detecting cervical carcinomas and advanced CIN2/3 lesions. Cancer Prev Res. 2014;7:1251-7.

8. Bierkens M, Hesselink AT, Meijer CJLM, Heideman DAM, Wisman GBA, Zee AGJ Van Der, et al. CADM1 and MAL promoter methylation levels in hrHPV-positive cervical scrapes increase proportional to degree and duration of underlying cervical disease. Int J Cancer.

2013;133:1293-300.

9. Eijsink JJH, Lendvai Á, Deregowski V, Klip HG, Verpooten G, Dehaspe L, et al. A four-gene methylation marker panel as triage test in high-risk human papillomavirus positive patients. Int J Cancer. 2012;130:1861-9.

10. Lorincz AT, Brentnall AR, Scibior-bentkowska D, Reuter C, Banwait R, Cadman L, et al. Validation of a DNA methylation HPV triage classifier in a screening sample. Int J Cancer. 2016;138:2745-51.

11. Hesselink AT, Heideman DAM, Steenbergen RDM, Coupé VMH, Overmeer RM, Rijkaart D, et al. Combined promoter methylation analysis of CADM1 and MAL: An objective triage tool for high-risk human papillomavirus DNA-positive women. Clin Cancer Res. 2011;17:2459-65.

12. Verhoef VMJ, Bosgraaf RP, Van Kemenade FJ, Rozendaal L, Heideman DAM, Hesselink AT, et al. Triage by methylation-marker testing versus cytology in women who test HPV-positive on self-collected cervicovaginal specimens (PROHTECT-3): A randomised controlled noninferiority trial. Lancet Oncol. 2014;15:315-22.

13. Snijders PJF, Steenbergen RDM, Heideman DAM, Meijer CJLM. HPV-mediated cervical carcinogenesis: Concepts and clinical implications. J Pathol. 2006;208:152-64.

14. Henken FE, Wilting SM, Overmeer RM, van Rietschoten JGI, Nygren AOH, Errami A, et al. Sequential gene promoter methylation during HPV-induced cervical carcinogenesis. $\mathrm{Br} J$ 
Cancer. 2007;97:1457-64.

15. Schütze DM, Kooter JM, Wilting SM, Meijer CJ, Quint W, Snijders PJ, et al. Longitudinal assessment of DNA methylation changes during HPVE6E7-induced immortalization of primary keratinocytes. Epigenetics. 2015;10:1-9.

16. Schutze DM, Krijgsman O, Snijders PJF, Ylstra B, Weischenfeldt J, Mardin BR, et al. Immortalization capacity of HPV types is inversely related to chromosomal instability. Oncotarget. 2016; doi: 10.18632/oncotarget.8058.

17. Steenbergen RDM, Ongenaert M, Snellenberg S, Trooskens G, Van Der Meide WF, Pandey D, et al. Methylation-specific digital karyotyping of HPV16E6E7-expressing human keratinocytes identifies novel methylation events in cervical carcinogenesis. J Pathol. 2013;231:53-62.

18. Bulkmans NWJ, Berkhof J, Rozendaal L, van Kemenade FJ, Boeke a JP, Bulk S, et al. Human papillomavirus DNA testing for the detection of cervical intraepithelial neoplasia grade 3 and cancer: 5-year follow-up of a randomised controlled implementation trial. Lancet. 2007;370:1764-72.

19. Steenbergen RD, Walboomers JM, Meijer CJLM, van der Raaij-Helmer EM, Parker JN, Chow LT, et al. Transition of human papillomavirus type 16 and 18 transfected human foreskin keratinocytes towards immortality: activation of telomerase and allele losses at 3p, 10p, 11q and/or 18q. Oncogene. 1996;13:1249-57.

20. Steenbergen RDM, Kramer D, Braakhuis BJM, Stern PL, Verheijen RHM, Meijer CJLM, et al. TSLC1 gene silencing in cervical cancer cell lines and cervical neoplasia. J Natl Cancer Inst. 2004;96:294-305.

21. van Zeeburg HJT, Snijders PJF, Pals G, Hermsen M a J a, Rooimans M a, Bagby G, et al. Generation and molecular characterization of head and neck squamous cell lines of fanconi anemia patients. Cancer Res. 2005;65:1271-6.

22. Jacobs M V, Snijders PJ, Brule AJC Van Den, Helmerhorst TJ, Meijer CJLM, Walboomers JM. A General Primer GP5+/GP6+-Mediated PCR-Enzyme Immunoassay Method for Rapid Detection of 14 High-Risk and 6 Low-Risk Human Papillomavirus Genotypes in Cervical Scrapings. J Clin Microbiol. 1997;35:791-5.

23. Hesselink AT, Berkhof J, van der Salm ML, van Splunter AP, Geelen TH, van Kemenade FJ, et al. Clinical validation of the HPV-risk assay, a novel real-time PCR assay for detection of high-risk human papillomavirus DNA by targeting the E7 region. J Clin Microbiol. 2014;52:890-6.

24. Mensaert K, Van Criekinge W, Thas O, Schuuring E, Steenbergen RDM, Wisman GBA, et al. Mining for viral fragments in methylation enriched sequencing data. Front Genet. 2015;6:16.

25. De Meyer T, Mampaey E, Vlemmix M, Denil S, Trooskens G, Renard J-P, et al. Quality evaluation of methyl binding domain based kits for enrichment DNA-methylation sequencing. PLoS One. 2013;8:e59068.

26. Langmead B, Trapnell C, Pop M, Salzberg SL. Ultrafast and memory-efficient alignment of short DNA sequences to the human genome. Genome Biol. 2009;10:R25.

27. Krueger F, Andrews SR. Bismark: A flexible aligner and methylation caller for Bisulfite-Seq applications. Bioinformatics. 2011;27:1571-2.

28. Overmeer RM, Henken FE, Snijders PJF, Claassen-Kramer D, Berkhof J, Helmerhorst TJM, et al. Association between dense CADM1 promoter methylation and reduced protein expression in high-grade CIN and cervical SCC. J Pathol. 2008;215:388-97.

29. Snellenberg S, De Strooper LMA, Hesselink AT, Meijer CJLM, Snijders PJF, Heideman DAM, et 
al. Development of a multiplex methylation-specific PCR as candidate triage test for women with an HPV-positive cervical scrape. BMC Cancer. 2012;12:551.

30. Thomas LK, Bermego JL, Vinokurova S, Jensen K, Bierkens M, Steenbergen RDM, et al. Chromosomal gains and losses in human papillomavirus-associated neoplasia of the lower genital tract - A systematic review and meta-analysis. Eur J Cancer. 2014;50:85-98.

31. Luttmer R, Strooper LMA De, Steenbergen RDM, Berkhof J, Snijders PJF, Heideman DAM, et al. Management of high-risk HPV-positive women for detection of cervical (pre)cancer. Expert Rev Mol Diagn. 2016;1-14.

32. De Meyer T, Bady P, Trooskens G, Kurscheid S, Bloch J, Kros JM, et al. Genome-wide DNA methylation detection by MethylCap-seq and Infinium HumanMethylation450 BeadChips: an independent large-scale comparison. Sci Rep. 2015;5:15375.

33. Bock C, Tomazou EM, Brinkman AB, Müller F, Simmer F, Gu H, et al. Quantitative comparison of genome-wide DNA methylation mapping technologies. Nat Biotechnol. 2010;28:1106-14.

34. Rodolakis A, Biliatis I, Symiakaki H, Kershnar E, Kilpatrick MW, Haidopoulos D, et al. Role of chromosome 3q26 gain in predicting progression of cervical dysplasia. Int J Gynecol Cancer. 2012;22:742-7.

35. Heselmeyer-Haddad K, Sommerfeld K, White NM, Chaudhri N, Morrison LE, Palanisamy N, et al. Genomic amplification of the human telomerase gene (TERC) in pap smears predicts the development of cervical cancer. Am J Pathol. 2005;166:1229-38.

36. Schneider KU, Dietrich D, Fleischhacker M, Leschber G, Merk J, Schäper F, et al. Correlation of SHOX2 gene amplification and DNA methylation in lung cancer tumors. BMC Cancer. 2011;11:102.

37. Kurscheid S, Bady P, Sciuscio D, Samarzija I, Shay T, Vassallo I, et al. Chromosome 7 gain and DNA hypermethylation at the HOXA10 locus are associated with expression of a stem cell related HOX-signature in glioblastoma. Genome Biol. 2015;16:16.

38. Boers A, Wang R, van Leeuwen RW, Klip HG, Bock GH De, Hollema H, et al. Discovery of new methylation markers to improve screening for cervical intraepithelial neoplasia grade $2 / 3$. Clin Epigenetics. 2016;8:29.

39. Ongenaert M, Wisman GBA, Volders HH, Koning AJ, van der Zee AGJ, Van Criekinge W, et al. Discovery of DNA methylation markers in cervical cancer using relaxation ranking. BMC Med Genomics. 2008;1:57.

40. Shi L, Du X, Jiang H, Xie J. Ghrelin and Neurodegenerative Disorders-a Review. Mol Neurobiol. 2016;doi:10.1007/s12035-016-9729-1.

41. Moskalev EA, Jandaghi P, Fallah M, Manoochehri M, Botla SK, Kolychev O V, et al. GHSR DNA hypermethylation is a common epigenetic alteration of high diagnostic value in a broad spectrum of cancers. Oncotarget. 2014;6:4418-27.

42. Grinberg I, Millen KJ. The ZIC gene family in development and disease. Clin Genet. 2005;67:290-6.

43. Qiang W, Zhao Y, Yang Q, Liu W, Guan H, Lv S, et al. ZIC1 is a putative tumor suppressor in thyroid cancer by modulating major signaling pathways and transcription factor FOXO3a. J Clin Endocrinol Metab. 2014;99:1163-72.

44. Huang RL, Gu F, Kirma NB, Ruan J, Chen CL, Wang HC, et al. Comprehensive methylome analysis of ovarian tumors reveals hedgehog signaling pathway regulators as prognostic DNA methylation biomarkers. Epigenetics. 2013;8:624-34. 
45. Gan L, Chen S, Zhong J, Wang X, Lam EKY, Liu X, et al. ZIC1 is downregulated through promoter hypermethylation, and functions as a tumor suppressor gene in colorectal cancer. PLoS One. 2011;6:e16916.

46. Wang LJ, Jin HC, Wang X, Lam EKY, Zhang J Bin, Liu X, et al. ZIC1 is downregulated through promoter hypermethylation in gastric cancer. Biochem Biophys Res Commun. 2009;379:95963.

47. Rai U, Thrimawithana T, Valery C, Young S. Therapeutic uses of somatostatin and its analogues: Current view and potential applications. Pharmacol Ther. 2015;152:98-110.

48. Jackson K, Soutto M, Peng D, Hu T, Marshal D, El-Rifai W. Epigenetic silencing of somatostatin in gastric cancer. Dig Dis Sci. 2011;56:125-30.

49. Jin Z, Mori Y, Hamilton JP, Olaru A, Sato F, Yang J, et al. Hypermethylation of the somatostatin promoter is a common, early event in human esophageal carcinogenesis. Cancer. 2008;112:43-9.

50. Mori Y, Cai K, Cheng Y, Wang S, Paun B, Hamilton JP, et al. A Genome-Wide Search Identifies Epigenetic Silencing of Somatostatin, Tachykinin-1, and 5 Other Genes in Colon Cancer. Gastroenterology. 2006;131:797-808.

51. Misawa K, Misawa Y, Kondo H, Mochizuki D, Imai A, Fukushima H, et al. Aberrant methylation inactivates somatostatin and somatostatin receptor type 1 in head and neck squamous cell carcinoma. PLoS One. 2015;10:e0118588.

52. den Boon JA, Pyeon D, Wang SS, Horswill M, Schiffman M, Sherman M, et al. Molecular transitions from papillomavirus infection to cervical precancer and cancer: Role of stromal estrogen receptor signaling. Proc Natl Acad Sci. 2015;112:E3255-64. 


\section{Tables}

Table 1. The selected 20 novel candidate methylation markers and the summarized results of the verification analyses.

\begin{tabular}{|c|c|c|c|c|}
\hline \multirow[b]{2}{*}{ Gene } & \multicolumn{2}{|c|}{ Verification by targeted bisulphite NGS } & \multicolumn{2}{|c|}{ Verification by MSP } \\
\hline & Cell lines & Tissues & Cell lines & Tissues \\
\hline$A N K R D 2$ & - & - & & \\
\hline CLDN18 & - & - & & \\
\hline GALR1 & + & + & + & \\
\hline GHSR & + & + & + & + \\
\hline GRK7 & - & - & & \\
\hline HIPK4 & - & - & & \\
\hline$I R X 1$ & + & + & + & \\
\hline MYCL2 & na & na & & \\
\hline NEUROG3 & + & + & + & \\
\hline PDE4DIP & + & na & & \\
\hline POU4F3 & + & + & + & + \\
\hline PROX1 & + & + & + & + \\
\hline RXFP3 & na & + & & \\
\hline SIM2 & + & + & + & \\
\hline$S L C 45 A 2$ & - & - & & \\
\hline soX14 & na & na & & \\
\hline SST & + & + & + & + \\
\hline TBX19 & - & - & & \\
\hline TFDP3 & - & - & & \\
\hline ZIC1 & + & + & + & + \\
\hline
\end{tabular}

+ verified differential methylation

- not verified differential methylation (i.e., detection of methylation in controls)

Highlighted in bold: 3 methylation markers selected for multiplex quantitative MSP

NGS: next generation sequencing; MSP: methylation-specific PCR; na: not assessable 
Table 2. Methylation level fold changes of GHSR, SST and ZIC1 per lesion category in cervical scrapes.

\begin{tabular}{|c|c|c|c|c|c|c|c|c|c|}
\hline \multirow{2}{*}{$\begin{array}{l}\text { A. } \\
\text { Category }\end{array}$} & \multicolumn{2}{|c|}{ Ct ratio GHSR } & \multicolumn{2}{|c|}{ CT ratio $S S T$} & \multicolumn{2}{|c|}{ CT ratio ZIC1 } & \multicolumn{3}{|c|}{ Fold changes over reference } \\
\hline & Median & Range & Median & Range & Median & Range & GHSR & SST & ZIC1 \\
\hline hrHPV+ control & 0.11 & $0-38.63$ & 0.06 & $0-19.09$ & 0.03 & $0-40.53$ & 1 (reference) & 1 (reference) & 1 (reference) \\
\hline CIN2 & 0.28 & $0-8.33$ & 0.06 & $0-3.19$ & 0.26 & $0-7.65$ & 2.50 & 1.09 & 10.08 \\
\hline CIN3 & 1.57 & $0.01-51.43$ & 0.65 & $0.01-27.56$ & 1.51 & $0.01-48.42$ & 14.07 & 11.05 & 58.66 \\
\hline SCC & 29.78 & $0.85-134.04$ & 9.13 & $0.06-69.81$ & 23.78 & $0.15-111.41$ & 266.06 & 154.91 & 921.12 \\
\hline B. & Ct ra & o GHSR & CT $r$ & io SST & CT $r$ & io ZIC1 & Fold ch & anges over re & ference \\
\hline Category & Median & Range & Median & Range & Median & Range & GHSR & SST & ZIC1 \\
\hline Early CIN2/3 & 0.14 & $0.04-0.78$ & 0.05 & $0-0.19$ & 0.02 & $0-0.16$ & 1.22 & 0.81 & 0.74 \\
\hline Advanced CIN2/3 & 1.49 & $0.07-26.58$ & 0.57 & $0-12.79$ & 1.22 & $0-26.24$ & 13.33 & 9.65 & 47.09 \\
\hline Without $3 q$ gain & 0.95 & $0.07-14.07$ & 0.13 & $0.01-5.26$ & 0.76 & $0-10.05$ & 8.47 & 2.28 & 29.60 \\
\hline With $3 q$ gain & 8.56 & $0.11-26.58$ & 3.00 & $0-12.79$ & 3.95 & $0.04-26.24$ & 76.42 & 50.90 & 153.04 \\
\hline
\end{tabular}

hrHPV+: high-risk human papillomavirus-positive; CIN: cervical intraepithelial neoplasia; SCC: squamous cell carcinoma 
Table 3. AUC, sensitivity and specificity of GHSR, SST and ZIC1 for endpoint CIN3+ in cervical scrapes.

\begin{tabular}{|l|c|c|c|c|}
\hline Methylation marker & AUC & Sensitivity (\%) & Specificity (\%) & p value $^{\text {a }}$ \\
\hline \hline GHSR & 0.87 & 76.5 & 83.5 & 0.000 \\
SST & 0.86 & 81.4 & 79.4 & 0.000 \\
ZIC1 & 0.89 & 86.3 & 80.4 & 0.000 \\
\hline
\end{tabular}

a value of the corresponding methylation marker in the univariable logistic regression model. AUC: area under the curve 


\section{Figures}

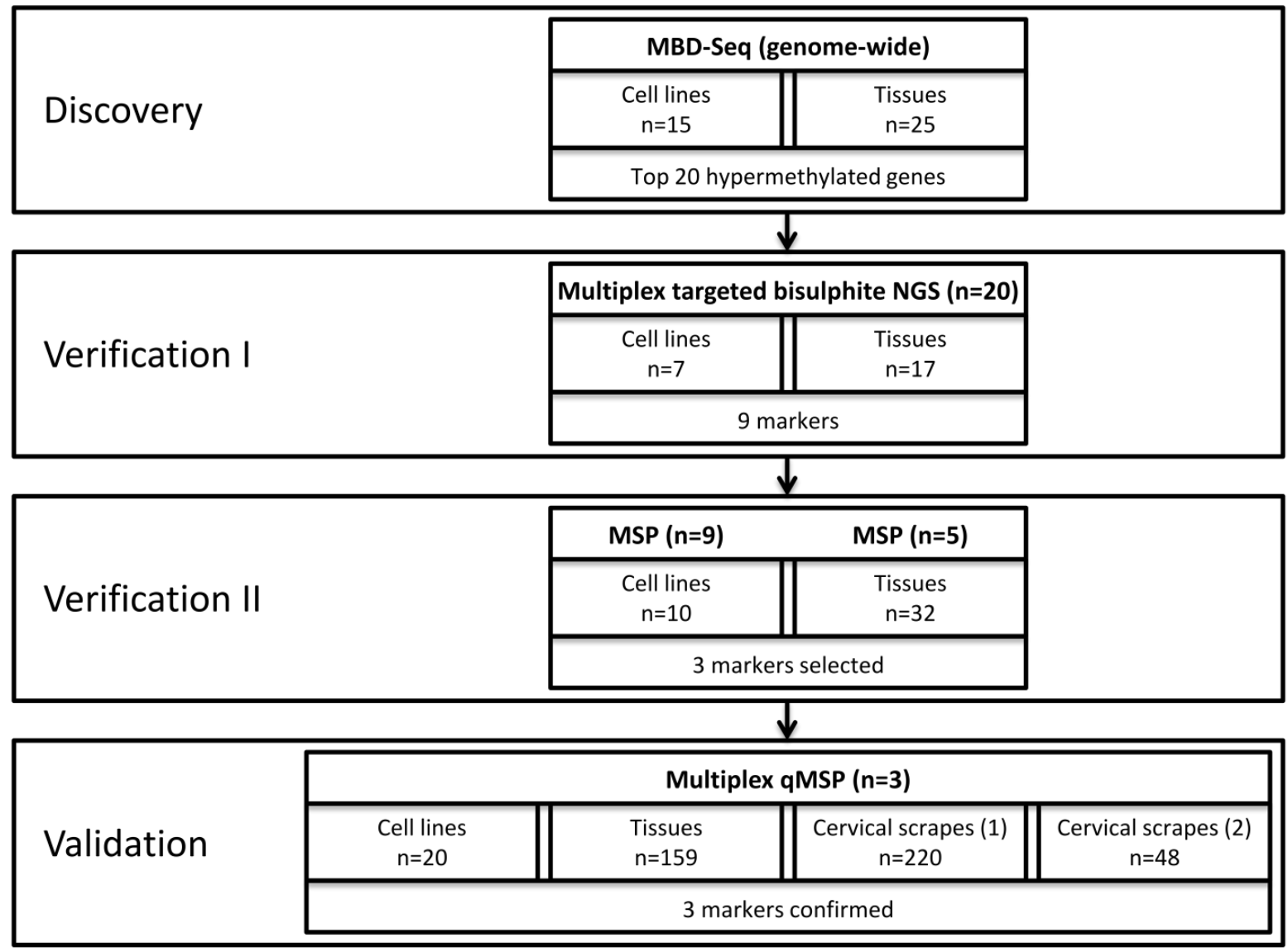

Figure 1. Experimental setup of the study. MBD-Seq: methyl binding domain; NGS: next generation sequencing; MSP: methylation-specific PCR; qMSP: quantitative MSP; (1) a cross-sectional series of hrHPV-positive cervical scrapes; (2) a series of hrHPV-positive cervical scrapes from women with an underlying $\mathrm{CIN} 2 / 3$ lesion and with a known duration of preceding hrHPV infection. 

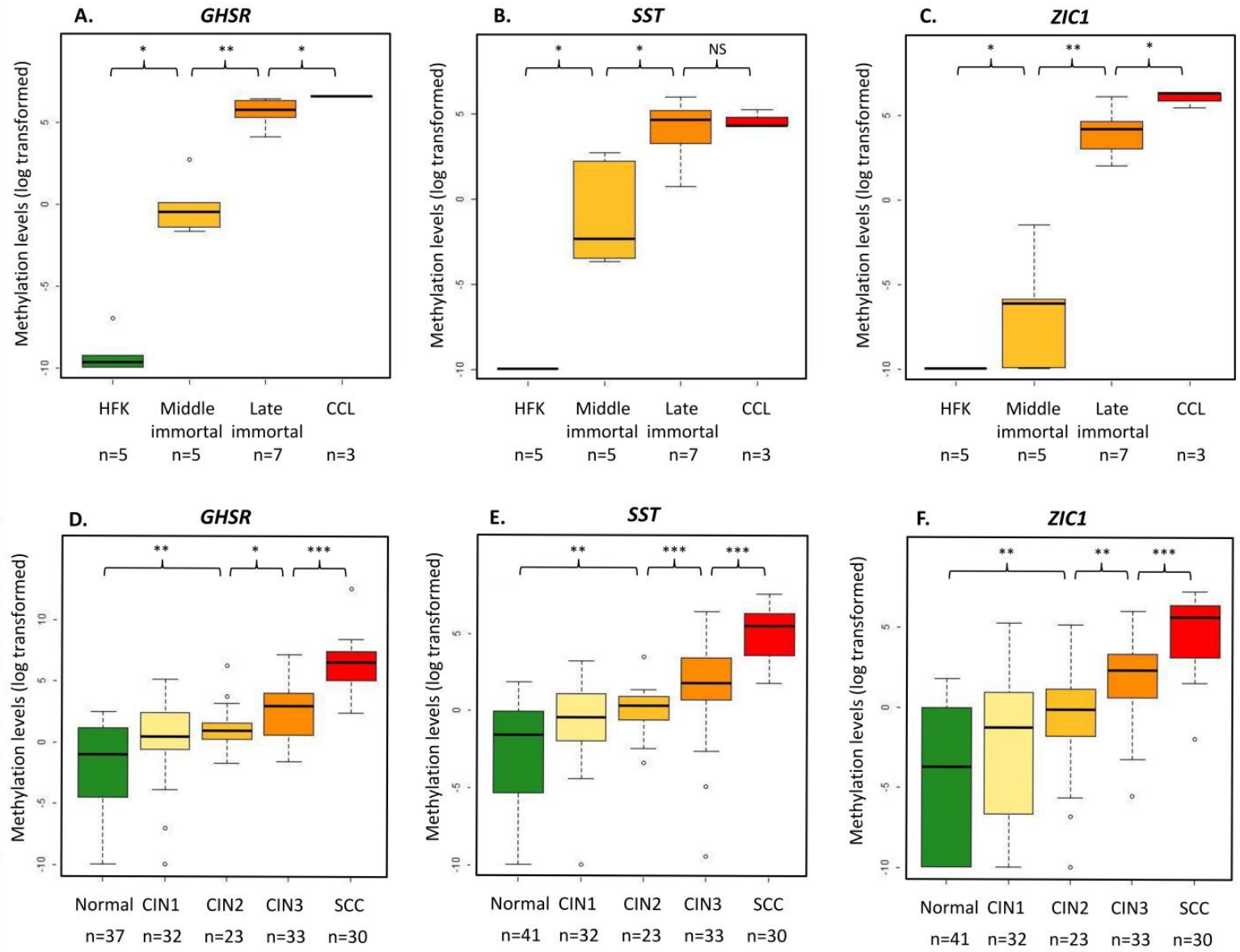

Figure 2. Validation of GHSR, SST and ZIC1 by qMSP in in vitro model and in cervical tissue specimens. Methylation levels of GHSR (A), SST (B) and ZIC1 (C) in in vitro model represented by the log2-transformed Cq ratios (y-axis) in the different groups of cell lines (x-axis). Methylation levels of GHSR (D), SST (E) and ZIC1 (F) in cervical tissue specimens represented by the log2-transformed Cq ratios (y-axis) in different histology groups (x-axis). HFK: human foreskin keratinocytes; middle immortal: FK16A/B and FK18A/B p30-p70; late immortal: FK16A/B and FK18A/B >p70; CCL: cancer cell lines; CIN: cervical intraepithelial neoplasia; SCC: squamous cell carcinoma.

${ }^{*} \mathrm{p}<0.05 ;{ }^{* *} \mathrm{p}<0.005 ;{ }^{* * *} \mathrm{p}<0.0005$; NS: not significant. 

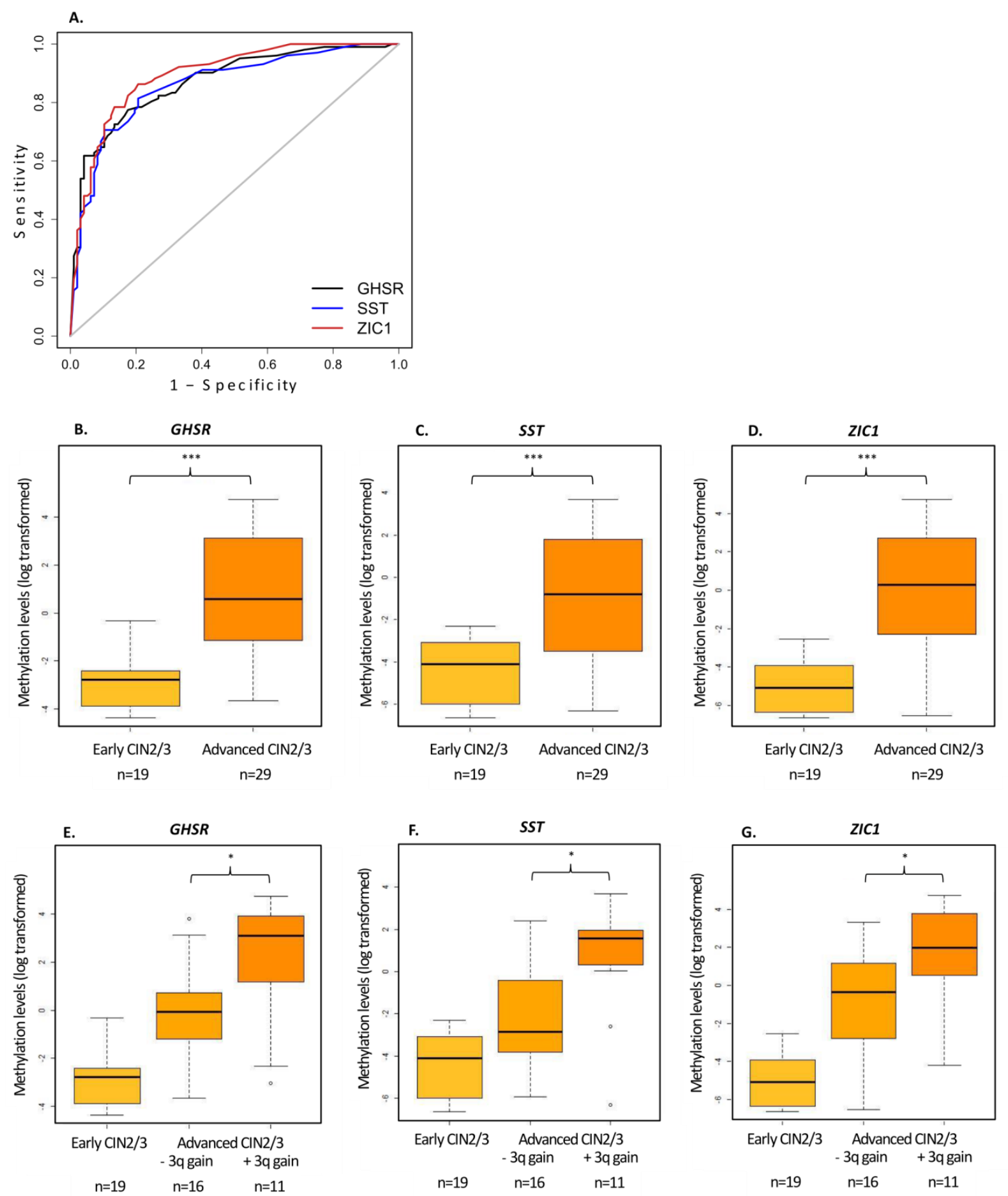

Figure 3. Validation of GHSR, SST and ZIC1 in hrHPV-positive cervical scrapes and their relation to duration of lesions existence and DNA copy number aberrations. Receiver operating characteristics (ROC) curves of GHSR, SST and ZIC1 for CIN3+ detection in hrHPV-positive cervical scrapes (A). Methylation levels of GHSR (B), SST (C) and ZIC1 (D) in hrHPV-positive cervical scrapes from women with $\mathrm{CIN} 2 / 3$ with a known duration of previous hrHPV infection (PHI), as a proxy for the duration of lesion existence; Early CIN2/3 corresponds to $<5$ year PHI; Advanced CIN2/3 corresponds to $\geq 5$ year PHI. Methylation levels of GHSR (E), SST (F) and ZIC1 (G) in hrHPV-positive cervical scrapes in relation to the presence of a chromosomal gain of respective chromosomal region (i.e., chr. 3q26, chr. 3q28 and chr. 3q24, respectively) in the underlying advanced $\mathrm{CIN} 2 / 3$ lesions. The $x$-axis displays the different groups (i.e., with (+3q gain) or without (- 3q gain) 3q gain) and the $y$-axis displays the log2transformed Cq ratios representing methylation levels. CIN: cervical intraepithelial neoplasia;

$* \mathrm{p}<0.05 ;{ }^{* * *} \mathrm{p}<0.0005$. 